\title{
Féeries
}

Études sur le conte merveilleux, XVII $-\mathrm{XIX}{ }^{\mathrm{e}}$ siècle

$10 \mid 2013$

Conte et croyance

\section{Les croyances en procès, la croyance en question : Diderot conteur}

Beliefs on Trial, Faith in Question: Diderot Tale-Teller

Jean-Christophe Igalens

\section{(2) OpenEdition}

1 Journals

Édition électronique

URL : http://journals.openedition.org/feeries/903

DOI : $10.4000 /$ feeries.903

ISSN : 1957-7753

Éditeur

UGA Éditions/Université Grenoble Alpes

\section{Édition imprimée}

Date de publication : 20 septembre 2013

Pagination : 253-272

ISBN : 978-2-84310-253-0

ISSN : 1766-2842

\section{Référence électronique}

Jean-Christophe Igalens, "Les croyances en procès, la croyance en question : Diderot conteur »

Féeries [En ligne], 10 | 2013, mis en ligne le 20 mars 2015, consulté le 08 septembre 2020. URL : http:// journals.openedition.org/feeries/903; DOI : https://doi.org/10.4000/feeries.903

\section{(c) Féeries}




\author{
Jean-Christophe Igalens \\ Université de Nice - Sophia Antipolis \\ Centre Transdisciplinaire d'Épistémologie de la Littérature
}

\title{
LES CROYANCES EN PROCĖS, LA CROYANCE EN QUESTION : DIDEROT CONTEUR
}

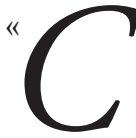

OMMENT S'Y PRENDRA DONC ce conteur-ci pour vous tromper ${ }^{\mathrm{I}}$ ?". Le «conteur historique», à la différence de ceux qui s'illustrent dans les contes merveilleux ou plaisants, veut être «cru» : en conclusion des Deux Amis de Bourbonne, Diderot expose les ressorts d'une "illusion" qui tient aux "petites circonstances». Cette illusion est produite par la "cicatrice légère ", la «verrue», la "coupure imperceptible à la lèvre» qui distinguent le personnage auquel le lecteur peut croire d'une figure «idéale». La réflexion sur le conte historique, "véridique et menteur», est une méditation sur les moyens d'entraîner l'adhésion à la fiction.

Dans Mystification, le philosophe se met en scène en commanditaire d'une tromperie. Il entend jouer sur la crédulité et les croyances de Mlle Dornet pour qu'elle se défasse des portraits de Galitzine, son ancien amant qui a épousé une autre femme. Il s'agira de lui faire "supposer à ces portraits une influence néfaste ${ }^{2}$ » pour l'effrayer. La chose semble aisée : "il y a un si grand fond à faire sur l'imagination d'une femme alarmée, et en général les femmes sont si crédules et pusillanimes en santé, si superstitieuses dans la maladie ${ }^{3}$ !» Un agent de change à la réputation douteuse, BonvaletDesbrosses, jouera le rôle d'un médecin turc adepte de la chiromancie et de l'onéirocritique ${ }^{4}$. Au-delà de l'ironie envers la supposée propension des femmes à la superstition, deux phénomènes attirent l'attention : les points de convergence entre le discours de l'imposteur et les préoccupations

I. Diderot, Les Deux Amis de Bourbonne, dans M. Delon (éd.), Contes et romans, avec la collaboration de J.-C. Abramovici, H. Lafon et S. Pujol, Paris, Gallimard, 2004, p. 449. Les citations de ce paragraphe renvoient à la même page.

2. Diderot, Mystification, dans Contes et romans, ouvr. cité, p. $4 \mathrm{I} 9$.

3. Ibid.

4. Soit la lecture de l'avenir dans les lignes de la main et l'interprétation des rêves comme présages. 
philosophiques de Diderot; la fin abrupte du conte qui se désintéresse du succès de la mystification, ce qui conduit à faire du moment de production de la croyance le véritable objet du texte. Si les croyances sont un enjeu thématique du conte, la croyance, ses ressorts, les moyens de l'obtenir intéressent plus encore Diderot conteur.

On peut en formuler l'hypothèse après ces deux brefs exemples : pour Diderot, le conte est peut-être moins une forme particulière qu'une façon de mettre à la fois en représentation et en question diverses facettes de la croyance $^{5}$ : adhésion fictionnelle, croyances au pluriel, disposition humaine fondamentale, fascinante et suspecte à la fois. Faire un acte de foi, devenir la dupe d'un imposteur, accepter des croyances superstitieuses, croire en une fiction sont des opérations différentes, mais qui engagent toutes, selon des modalités diverses, des effets de croyance. Après tout, écrit Diderot, le conteur historique cherche bien à "tromper" son lecteur, c'est-à-dire qu'il n'y a pas nécessairement, ou pas immédiatement, solution de continuité, sous sa plume, entre la croyance sérieuse et l'adhésion fictionnelle : cette différence, cet écart, ou la relation qui unissent les deux formes de croyance restent une question à poser, un problème à penser, dans des termes qui ne sont pas nécessairement ceux des théories modernes de la fiction ${ }^{6}$. L'écriture diderotienne du conte serait ainsi tendue entre critique

5. Sur le problème de savoir ce qui, chez Diderot, relève du «conte» ou d'autres formes littéraires, nous adoptons volontiers la démarche pragmatique et ouverte suggérée notamment par Jean Ehrard ("Quand il s’agit de cet inclassable Diderot cessons donc de jouer les Linné de la littérature, renonçons à étiqueter, laissons-nous prendre à cette surprenante tératologie littéraire, pour le bonheur de l'intelligence, pour le plaisir ", "Diderot conteur (2) : l'art de déplacer la question", dans J. Ehrard, L'Invention littéraire au XVIII siècle : fictions, idées, société, Paris, PUF, I997, p. I63-173, p. 173) et Michel Delon ("Aucun classement de ses ouvrages ne pourra prétendre à un ordre définitif, le travail de lecture doit prolonger le travail d'écriture dans l'hybridation formelle et la circulation conceptuelle. [...] L'exemple de l'ami Denis doit inviter à une modestie joyeuse et à ce qu'on pourrait appeler un opportunisme critique», dans Diderot, Contes et romans, ouvr. cité, "Préface», p. xxix). L’enquête a évidemment inclus Les Deux Amis de Bourbonne et la série de textes présentés comme des "contes" par la Correspondance littéraire, Ceci n'est pas un conte, Madame de La Carlière et le Supplément au voyage de Bougainville, ainsi que L'Oiseau blanc, conte bleu. L'Entretien d'un père avec ses enfants, qui fut publié dans les Contes moraux et nouvelles idylles de D... et Salomon Gessner en I773 a aussi été pris en considération, comme Mystification. On ne pouvait pas ne pas avoir La Religieuse à l'esprit, dans la mesure où sa relation avec la mystification relie cette ouvre aux Deux Amis de Bourbonne et à Mystification. Ont donc été privilégiés des textes désignés, d'une façon ou d'une autre, comme des "contes" (ce qui est après tout aussi le cas de La Religieuse, par le biais de la locution «faire un conte», nous le rappellerons) et caractérisés par une certaine brièveté, sans que l'on se soit interdit de mettre ces textes en relation avec d'autres, ni prétendre en sens inverse avoir couvert l'ensemble de ces relations possibles. On verra que Les Deux Amis de Bourbonne, Madame de La Carlière et Mystification occupent une place privilégiée dans la lecture que nous proposons.

6. Voir sur ce point, pour une analyse des différences entre la feintise sérieuse et la feintise ludique partagée et sur l'immersion fictionnelle, J.-M. Schaeffer, Pourquoi la fiction?, Paris, Seuil, 
des croyances et analyse de la croyance, entre recherche d'une adhésion à la fiction pensée sur le modèle de la croyance sérieuse et mise à jour ludique du fonctionnement même de cette croyance.

Les contes de Diderot peuvent parfois souligner plaisamment l'absurdité intrinsèque de croyances religieuses. Dans L'Oiseau blanc, conte bleu, le pouvoir du Saint-Esprit et la virginité de Marie sont ainsi tournés en dérision. L'oiseau blanc a charmé par son chant des vierges enfermées dans un cloître : prodige, «il en naquit nombre de petits esprits, sans que la virginité de ces filles en souffrît ${ }^{7}$ ». La sultane refuse cette partie du récit : «Je veux bien qu'on me fasse des contes, mais je ne veux pas qu'on me les fasse aussi ridicules ${ }^{8}$.» Le trait d'esprit consiste à faire de l'objet de foi identifié par le lecteur une péripétie irrecevable au sein du conte merveilleux où pourtant, selon la définition de Diderot, «rien ne se passe comme dans [la terre] que vous habitez», la nature y étant "exagérée» et la vérité "hypothétique ${ }^{9}$. Le dogme religieux passe les bornes du merveilleux, ce qui le disqualifie. La croyance qu'il suscite est en-deçà de l'adhésion sollicitée par cette sorte de contes.

Cette critique conventionnelle n'est pas la plus essentielle. Le contenu des croyances, s'il demeure obliquement visé, importe moins par lui-même qu'en raison de son influence sur le jugement moral. Orou s'adresse à l'aumônier du Supplément au voyage de Bougainville: "Je ne sais ce que c'est que ton grand ouvrier, mais je me réjouis qu'il n'ait point parlé à nos pères, et je souhaite qu'il ne parle point à nos enfants, car il pourrait par hasard leur dire les mêmes sottises, et ils feraient peut-être celle de les $\operatorname{croire}^{\mathrm{IO}}$." Cet acte de foi serait nuisible parce que les croyances qui en résultent contredisent la loi naturelle : le conte, censé délivrer, selon la Correspondance littéraire, "la morale et le but secret ${ }^{\mathrm{II}} »$ de deux autres, Ceci n’est pas un conte et Madame de la Carlière, traite en particulier de la sexualité, mais la leçon est plus générale. À la relativité des croyances s'oppose l'universalité de la loi naturelle; les premières font courir le risque de s'éloigner de la seconde, d'en faire taire la voix.

1999. Nous avons préféré ne pas partir d'une définition théorique moderne de ces formes de feintise et du type de "croyance » qu'elles peuvent solliciter, pour ne pas répondre a priori aux questions que soulèvent les textes de Diderot.

7. Diderot, Euvres, t. III, édition établie par L. Versini, Paris, Robert Laffont, 1994, p. 225.

8. Ibid.

9. Diderot, Les Deux Amis de Bourbonne, ouvr. cité, p. 448.

Io. Diderot, Supplément au voyage de Bougainville, dans Contes et romans, ouvr. cité, p. 556.

II. Voir les notices de M. Delon et de S. Pujol dans Diderot, Contes et romans, ouvr. cité, p. I079, n. 4 et p. 1098. 
Cette critique s'entendait dans les contes avant le triptyque dont le Supplément fournit la «morale ${ }^{\mathrm{I2}} »$. Dans l'Entretien d'un père avec ses enfants, Moi s'indigne de l'influence du père Bouin qui, en faisant référence à la Providence, convainquit son père de refuser un héritage à de pauvres gens au profit d'un homme riche et antipathique sur la foi d'un testament douteux : "il fallait écouter votre cœur qui n’a cessé de réclamer depuis, et qui en savait plus que votre imbécile Bouin dont la décision ne prouve que l'autorité redoutable des opinions religieuses sur les têtes les mieux organisées ${ }^{13}$ ».

Les Deux Amis de Bourbonne, dans leur seconde version, abordent la même question par la structure du conte. Celle-ci se fonde sur un principe d'emboîtement. Les premières pages, qui rapportent l'histoire d'Olivier et Félix, relèvent de la fiction épistolaire. Celle-ci, plus nettement marquée dans la première version, est maintenue dans la seconde : le narrateur écrit à "petit frère». Selon la genèse du conte, le narrateur est en réalité une narratrice, et le "petit frère" est identifié à Naigeon. Le texte peut cependant se lire sans ces explicitations. Deux lettres se succèdent, sans solution de continuité ${ }^{14}$ : la première contient l'histoire des deux amis, la seconde, répondant à une sollicitation du destinataire qui a réclamé la suite des aventures de Félix après la mort d'Olivier, en contient une autre, qui à son tour en inclut une nouvelle. La première lettre "enchâssée», écrite par le subdélégué Aubert, rapporte les aventures de Félix. La seconde, beaucoup plus brève, transmise par le subdélégué, est due à la plume du curé Papin qui porte un jugement moral négatif sur les deux amis et la veuve d'Olivier. Les dernières lignes du conte ne reviennent pas au premier plan énonciatif : une nouvelle voix se fait entendre. Jean Ehrard interprétait certaines "contradictions du récit» comme un "sourire de malice et de complicitét ${ }^{15}$ » adressé au lecteur vigilant. Cette description de la relation de Diderot avec son lecteur est profondément juste, mais une des

I2. Jean Ehrard a souligné que l'unité des trois contes ne tient pas seulement au matériel thématique : elle réside "dans la manière personnelle dont Diderot combine ici la narration et le dialogue, dans la cohérence d'une structure dynamique, génératrice d'un fort mouvement de pensée» ("Diderot conteur (2) : l'art de déplacer la question", art. cité, p. I64).

13. Diderot, Entretien d'un père avec ses enfants, dans Contes et romans, ouvr. cité, p. 484.

I4. Cela est encore plus net dans les manuscrits qui ne comportent pas l'épisode intercalé du lieutenant de Testalunga. La Correspondance littéraire par exemple, qui explicite par ses notes la fiction épistolaire "cadre», ne contient pas ce récit : son hétérogénéité énonciative — ce n'est plus le même narrateur qui raconte - est assez nette. En tant que rupture de la fiction épistolaire "cadre», elle annonce celle que nous allons rencontrer à la fin du conte.

I5. J. Ehrard, «Diderot conteur (I) : la subversion du conte moral», dans J. Erhard, L'Invention littéraire au XVIII e siècle, ouvr. cité, p. I60, 
contradictions que Jean Erhard relevait n'en est sans doute pas une. La «situation économique de la veuve d'Olivier ${ }^{16}$ » serait présentée de façon contradictoire : "Nous avons parlé d'elle, et j'espère que notre recommandation ne lui aura pas été inutile ${ }^{17}$ ", lit-on au premier niveau énonciatif. La fin du conte rapporte l'effet du jugement du curé Papin par une dernière lettre prouvant que l'homme d'Église a été écouté, suivie par une conclusion du narrateur :

On pense bien que la veuve Olivier et Félix n'eurent aucune part aux aumônes de Mme de $e^{* * *}$. Félix mourut; et la pauvre femme aurait péri de misère avec ses enfants, si elle ne s'était réfugiée dans la forêt chez son fils aîné où elle travaille, malgré son grand âge, et subsiste comme elle peut, à côté de ses enfants et de ses petits-enfants ${ }^{18}$.

La lettre du curé Papin et la réponse qu'il reçoit sont nécessairement ultérieures au commentaire optimiste du premier narrateur — l'épistolière. La fin du texte montre que cette dernière s'est trompée, et plus encore, que l'un des personnages inclus dans son "nous», "maman», a changé d'avis sur les deux amis et la veuve après la lettre du curé. Il y a donc moins une incohérence narrative qu'une rupture énonciative, également perceptible dans la modification de la façon dont est désignée la destinataire des lettres du subdélégué Aubert et du curé : «maman » sous la plume de la première épistolière, “Mme de*** » à la fin du texte. Ce n'est plus le même narrateur qui raconte. Et ce glissement a une fonction narrative : l'histoire complète n'est pas seulement celle des deux amis, puis de Félix et de la veuve, suivie d'une conclusion dysphorique hétérogène, elle intègre aussi le renversement de l'évaluation morale, l'abolition de «l'intérêt" pris à ces personnages par un autre personnage, $M m e \mathrm{de}^{* * *}$, sous l'influence d'«opinions religieuses».

L'histoire du texte peut inviter à lire les lettres du subdélégué et du curé comme un diptyque : la première version du conte ne comportait qu'une lettre, celle de Papin, qui contenait à la fois l'histoire de Félix et le jugement moral du prêtre. Désormais le récit — et l'intérêt au sens classique qu'il suscite pour les personnages - ainsi que le jugement sont confiés à deux instances qui se contredisent ${ }^{19}$. Mais en réalité, la lettre

I6. Ibid., note 3 .

17. Diderot, Les Deux Amis de Bourbonne, ouvr. cité, p. 44I.

I8. Ibid., p. 448.

19. Selon Jean Varloot, cette modification et les transformations qui lui sont associées modifient l'image du curé, uniquement "intéressé et hostile aux gueux» dans la seconde version (J. Varloot, "Les Deux Amis de Bourbonne. Une version originale fort signifiante", Revue de la Bibliothèque nationale, I7, 1985, p. 49). Si le personnage du curé n'était manifestement pas fait, dès la première 
de Papin s'oppose aux deux premiers plans énonciatifs, aux deux récits qui conduisent à chaque fois à prendre le parti des personnages humbles, révoltés, malheureux et vertueux. Sa brièveté même est une illustration de «l'influence redoutable» des «opinions religieuses» dénoncée par Moi dans l'Entretien d'un père avec ses enfants. L'ultime plan énonciatif produit un effet de chute : alors que le lecteur a été conduit à adopter une attitude empathique à l'égard des pauvres, il apprend en quelques lignes que les références à la "Providence», au «respect dû à l'Église et à ses ministres ${ }^{20}$ " ont suffi à altérer le jugement de Mme de ${ }^{* * * 21}$.

Ces emboîtements énonciatifs ont la particularité de ne faire apparaître le narrateur «premier» qu'à la fin du conte : tout se passe comme si Diderot faisait tomber au dernier moment le masque de la fiction épistolaire, censée accréditer les événements rapportés, pour faire entendre la voix d'un conteur omniscient qui transforme la première narratrice et "maman" en personnages du conte. Ce principe d'enchâssement non seulement de lettres, mais de plans énonciatifs forme un système avec «la chaîne des témoignages" repérée et analysée par Pierre Chartier ${ }^{22}$. La première épistolière tient l'histoire des deux amis de la veuve d'Olivier; elle transmet celle de Félix par l'intermédiaire du subdélégué Aubert qui l'a apprise par la charbonnière. La répétition de cette formule d'attestation chez l'épistolière et sous la plume du subdélégué est un de ces jeux retors et ironiques par lesquels Diderot, d'un même mouvement, construit et déconstruit un effet de croyance : " [Le subdélégué Aubert] nous a envoyé le récit suivant, sur la vérité duquel vous pouvez compter» écrit la première; et le second s'adresse à Mme de*** en ces termes : "Je vous conjure, Madame, de vous en tenir aux faits, sur la vérité desquels vous pouvez compter ${ }^{23}$." Le témoignage d'autrui, s'il faut suivre les considérations de Hume dans le Traité sur la nature humaine, est un puissant vecteur de croyance : Hume souligne notamment l'influence

version, pour attirer la sympathie du lecteur, il semble en effet que la radicalisation de ses traits les plus négatifs dans la seconde version participe au dispositif énonciatif que nous essayons d'analyser. 20. Ainsi qu'à la loi : on retrouve ici la problématique de l'Entretien d'un père avec ses enfants.

2I. Il faut noter que le discours du curé, évidemment discrédité par ses propres termes ("qu’est-ce aux yeux de Dieu que la plus sublime vertu dénuée des sentiments de la piété, du respect dû à l'Église [...]", Les Deux Amis de Bourbonne, ouvr. cité, p. 447), dénonce sa propre contradiction avec l'Évangile : «L'Évangile ne cesse de nous recommander la commisération pour les pauvres; mais on double le mérite de sa charité par un bon choix des misérables, et personne ne connaît mieux les vrais indigents que le pasteur commun des indigents et des riches.» (Ibid.)

22. P. Chartier, "Parole et mystification. Essai d'interprétation des Deux Amis de Bourbonne», dans J. Proust (dir.), Recherches nouvelles sur quelques écrivains des Lumières, Genève, Droz, 1972, p. 203-27I, p. 246.

23. Les Deux Amis de Bourbonne, ouvr. cité, p. 44I et 446. Nous soulignons. 
de la ressemblance dans la croyance qu'il aborde, de façon nouvelle, comme une opération de l'esprit. Son propos sur le témoignage est aussi une critique de la crédulité, ce qui est, nous le verrons, un thème explicite de Madame de La Carlière. Mais la visée démystificatrice et sceptique n'est pas le seul enjeu des pages que le Traité de la nature humaine consacre à la croyance ${ }^{24}$ : c'est au fonctionnement d'une opération de l'esprit cruciale que se consacre le philosophe.

Les autres effets, écrit-il, n'indiquent leur cause que d'une manière oblique; mais le témoignage des hommes le fait directement, et l'on doit le considérer comme une image aussi bien que comme un effet. Il n'y a donc pas à s'étonner que nous soyons si prompts à en tirer des inférences ${ }^{25}$.

Si on lit Les Deux Amis de Bourbonne à la lumière de ce développement, le recours à l'autorité du témoignage n'est pas en lui-même un indice invitant le lecteur à la vigilance : il peut au contraire se fonder sur un fonctionnement de l'esprit en vertu duquel le témoignage, véritable image, est susceptible de donner aux idées ou aux faits qu'il rapporte le degré de vivacité distinguant les choses auxquelles on croit et celles que l'on peut concevoir sans leur accorder crédit ${ }^{26}$. En revanche, l'enchâssement des témoignages, sur lequel Diderot attire l'attention par la répétition de la formule d'attestation, a tout d'un signal invitant le lecteur à prendre conscience du fonctionnement de sa propre croyance : le conteur rappelle la présence de trois filtres (la charbonnière, le subdélégué, l'épistolière) entre le destinataire du conte et les «faits» qui semblent pourtant s'offrir en toute transparence.

Que cette construction intervienne à propos des récits qui sont censés susciter l'intérêt pour les deux amis et la veuve n'est pas un paradoxe. L'enchâssement des niveaux énonciatifs représente et rend sensible l'effet pernicieux des opinions religieuses sur le jugement. Diderot ne se contente pas d'opposer à cet effet une démystification rationnelle ou un autre effet de croyance, plus juste quant à son contenu, mais fondé sur la même opération de l'esprit. Il entremêle les deux démarches : il invente un conte qui, pour emporter l'adhésion du lecteur, représentée dans le texte par la

24. D. Hume, L'Entendement. Traité de la nature humaine. Livre I et appendice, traduction par P. Baranger et P. Saltel, Paris, GF Flammarion, 1995, troisième partie, sections VII à XI.

25. Ibid., p. I80.

26. Voir par exemple ibid., p. I62 : «Mais la croyance est un peu plus qu'une simple idée. C’est une manière particulière de former une idée, et puisque la même idée ne peut être modifiée que par une modification de ses degrés de force et de vivacité, il s'ensuit, finalement, que la croyance est une idée vive produite par une relation avec une impression présente.» 
première réaction de $\mathrm{Mme} \mathrm{de}^{* * * 27}$, doit produire l'illusion théorisée à la suite des Deux Amis; et, d'un même élan, il montre le fonctionnement de cette illusion, non seulement dans les considérations théoriques conclusives, mais aussi dans le conte lui-même, par le travail de "dénonciation du leurre au moment même où il est énoncé ${ }^{28}$ " qu'analyse Pierre Chartier. Jean Ehrard relève que le «suffisant lecteur» des Deux Amis de Bourbonne, celui auquel Diderot adresse ses sourires, est «capable de compatir à la triste histoire qui lui est contée tout en gardant la tête froide et l'esprit critique éveillé2 ${ }^{2} »$. Cette double disposition, ce paradoxe sur le lecteur, se fonde sur l'articulation d'une dénonciation de mauvaises croyances et d'un jeu beaucoup plus complexe sur les mécanismes de la croyance : sollicités pour produire l'empathie et l'intérêt, pour le plaisir spécifique qu'ils procurent, ils sont mis à distance afin que le conteur ne devienne pas un «trompeur" comme les autres qui priverait le lecteur de son autonomie de jugement, fût-ce pour la bonne cause ${ }^{30}$.

Ce mouvement est redoublé par les liens que le conte, comme d'autres fictions narratives de Diderot, entretient avec la mystification. Toutes ces fictions n'ont pas un statut identique : "L'Histoire des portraits, note Jacques Proust, est le journal d'une mystification, alors que La Religieuse est la suite d'une mystification, dont la Préface-Annexe est le support. Les Deux

27. «Je vous avoue que l'histoire de ces deux hommes m'avait touchée; et vous conviendrez que l'exemple d'une amitié si rare, était bien fait pour séduire une âme honnête et sensible. " (Les Deux Amis de Bourbonne, ouvr. cité, p. 448.)

28. P. Chartier, "Le conte "historique". Diderot théoricien de la mystification dans Les Deux Amis de Bourbonne", dans D. Gallingani (dir.), Le credibili finzioni della storia, Florence, Central editoriale Toscano, I996, p. Io.

29. J. Ehrard, «Diderot conteur (I) : la subversion du conte moral», art. cité, p. I60.

30. Pierre Chartier met l'accent sur cette visée émancipatrice et politique du va-et-vient entre mystification et démystification, au premier chef dans Les Deux Amis de Bourbonne : son objet serait « la dénonciation folle ou rieuse, mais c'est tout un, d'un acte de foi ou de superstition que l'“autorité" se flatte d'imposer à nos âmes pusillanimes. Mystifier, puis démystifier, c'est donc garantir notre "autonomie", c'est préserver en chaque lecteur sa liberté de sujet responsable, de membre de plein droit du tribunal de l'opinion publique» ("Diderot, ou le rire du mystificateur», Dix-huitième siècle, 32, 2000, p. I63). Michel Delon souligne la valeur positive des jeux mêmes de la croyance, qui ne seraient pas seulement le moment négatif du mouvement dialectique vers l'émancipation : «Le philosophe athée sait que l'homme se nourrit de songes autant que d'éléments matériels, que ses désirs ont besoin d'imagination autant que de réalité, mais si la religion impose des fictions qui se prétendent des vérités transcendantes, le roman peut concilier la fiction et la lucidité [...]. Tout lecteur, ajoute Diderot [à Cervantès], a besoin d'illusions et ce n'est pas en brûlant les livres qu'on apaisera ce besoin, c'est en le satisfaisant par l'entraînement narratif et en empêchant que l'illusion devienne folie romanesque ou manipulation entre les mains de ceux qui y ont intérêt. Aux égarements du cœur et de l'esprit, causés par le mauvais romanesque, le philosophe oppose le double plaisir de la sympathie et de l'intelligence, de l'émotion et de l'ironie.» ("Préface», dans Diderot, Contes et romans, ouvr. cité, p. xxxii-xxxiii.) 
Amis, comme la Préface-Annexe de La Religieuse et sa suite romanesque constituent aussi, en eux-mêmes, une mystification ${ }^{31}$. " À propos des Deux Amis de Bourbonne, on peut ajouter que le lecteur, pas plus aujourd'hui que du temps de Diderot, ne lit le texte qui servit à mystifier Naigeon. En revanche, cette mystification réelle est rappelée par Grimm dans la Correspondance littéraire ${ }^{32}$, comme elle l'est dans les éditions modernes : l'efficacité mystificatrice du conteur est inscrite dans le péritexte et participe à la réception. Le lecteur est censé lire un texte qui a été cru, que son premier destinataire a pris pour vrai : lui ne sera pas dupe, mais, d'un même mouvement, il est invité à faire de la possibilité de croire ce qu'il lit une composante de la lecture. Situation au fond symétriquement inverse du jeu habituel produit par les préfaces de roman qui garantissent la vérité du récit sans que le lecteur soit dupe : ici, par la présentation de Grimm puis en vertu des réflexions de Diderot à la fin du texte, l'œuvre se présente comme un leurre, ou comme le prolongement d'un leurre, qui ne doit donc plus en être un pour le lecteur, mais qui l'a été efficacement pour autrui. La question se déplace de la production vers la réception, de la "vérité», tant de fois alléguée qu'elle est devenue une "convention" fictionnelle, vers la croyance.

Quoique Diderot se fonde sur des mystifications réelles, Les Deux Amis de Bourbonne, comme le péritexte de La Religieuse, peuvent représenter une transformation littéraire sophistiquée (et assez machiavélique) des préfaces romanesques construisant une fiction censée accréditer la fiction principale (manuscrit trouvé et autres correspondances dévoilées) : "dénoncer» in fine la fiction comme un mensonge sérieux, ou comme sa réélaboration, c'est encore l'insérer, au second degré, dans une histoire vraie, celle de l'auteurmystificateur. Le récit n'est plus l'image ou la représentation d'une histoire vraie, mais il en constitue malgré tout une trace, effet ou conséquence. Situation retorse, dont l'ensemble des effets reste peut-être encore à analyser. On relèvera simplement qu'elle substitue à la pseudo-garantie de vérité une "authentique» garantie de croyance : le conte n’est pas vrai, mais on peut véritablement y croire. En «dévoilant» les mécanismes de l'illusion dans Les Deux Amis de Bourbonne et en rappelant l'origine de l'œuvre dans La Religieuse, dans les deux cas après le récit, Diderot invite le lecteur à se distancier de

3I. J. Proust, «Introduction», dans Diderot, Quatre contes, Jacques Proust (éd.), Genève, Droz, I964, p. xii. Sur les relations entre Diderot et la mystification, voir également J. Catrysse, Diderot et la mystification, Paris, Nizet, 1970 ainsi que les articles cités de Pierre Chartier.

32. «[Le philosophe] fit quelques contes que la jeune amie malade inséra dans ses lettres à son ami crédule qui les prit pour des faits avérés.» ("Présentation par Grimm [...] dans la Correspondance littéraire (Is décembre 1770)", dans Diderot, Contes et romans, ouvr. cité, p. 463.) 
la dupe par une démarche démystificatrice : comme l'écrit Michel Delon, il concilie un paradoxe "en perfectionnant l'illusion et dévoilant ses trucs, en mystifiant son lecteur pour le démystifier ensuite ${ }^{33} »$. Le moment de la croyance (possible) demeure cependant fondamental, comme le montre la légende auctoriale que Diderot laisse figurer dans la préface-annexe de $\mathrm{La}$ Religieuse. L'auteur-mystificateur redoute une extension de la mystification dont il deviendrait lui-même la victime ${ }^{34}$, mais il est aussi capable de "croire" à son propre conte, pourvu qu'il en retire un bénéfice d'émotion :

Une circonstance qui n'est pas la moins singulière, c'est que, tandis que cette mystification échauffait la tête de notre ami en Normandie, celle de M. Diderot s'échauffait de son côté. Celui-ci, persuadé que le marquis ne donnerait pas un asile dans sa maison à une jeune personne sans la connaître, se mit à écrire en détail l'histoire de notre religieuse. Un jour qu'il était tout entier à ce travail, M. d'Alainville, un de nos amis communs, lui rendit visite, et le trouva plongé dans la douleur et le visage inondé de larmes. "Qu’avez-vous donc? lui dit M. d'Alainville. Comme vous voilà! — Ce que j’ai? lui répondit M. Diderot; je me désole d'un conte que je me fais ${ }^{35}$.

Faire un conte désigne ici une activité d'imagination par laquelle le mystificateur invente une histoire qu'il ne s'agit pas exactement de croire comme un fait, mais qui doit entraîner une adhésion suffisante pour échauffer la tête, "faire frissonner la peau et couler les larmes ${ }^{36}$ ». La croyance, à lire Hume, possède sur les autres façons de considérer une idée ou un fait un privilège d'intensité : le lecteur qui croit à ce qu'il lit «a une conception plus vive de tous les événements, il entre plus profondément dans l'intérêt des personnages, il se représente leurs actions, leurs caractères, leurs amitiés et leurs inimitiés; il va même jusqu'à se faire une idée de leurs traits, de leur air et de leur personne ${ }^{37}$ ». La croyance est ainsi efficace : «l'effet de la croyance est donc d'élever une simple idée jusqu'à l'égalité avec nos impressions, et de lui conférer une influence similaire sur les passions. [...] [L] a croyance est presque absolument requise pour susciter nos passions ${ }^{38}$.» On formulera ici une hypothèse, sans entrer pour autant dans un débat sur les sources philosophiques du conteur : une telle conception de la croyance, fondée

33. M. Delon, «Préface», dans Diderot, Contes et romans, ouvr. cité, p. xxxiii.

34. Diderot, La Religieuse, «Préface-annexe», dans Contes et romans, ouvr. cité, p. 385 : «les lettres du généreux protecteur de la recluse sont véritables et ont été écrites de bonne foi, ce qu’on eut toutes les peines du monde à persuader à $\mathrm{M}$. Diderot qui se croyait persiflé par le marquis et par ses amis".

35. Ibid., p. 384 .

36. Les Deux Amis de Bourbonne, ouvr. cité, p. 449.

37. Hume, Traité de la nature humaine, ouvr. cité, p. I62.

38. Ibid., p. I87-I88. 
sur la vivacité avec laquelle l'idée ou le fait sont considérés, n'est sans doute pas étrangère au fait que le conteur veuille être " $\mathrm{Cru}^{39}$ ", que Diderot préfère "l'illusion ${ }^{40}$ " produite par le texte à l' "admiration" que pourrait susciter un auteur exhibant ses effets.

Les trois opérations des Deux Amis de Bourbonne (la critique narrative de l'autorité des opinions religieuses, la production d'une histoire émouvante pouvant susciter la croyance et la déconstruction de cette seconde adhésion) sont indissociables : le conte est le fruit de leurs relations. Les croyances religieuses ne sont d'ailleurs pas les seules contre lesquelles le lecteur se trouve mis en garde. Au sein de la chaîne "polémique» ou «critique» des témoignages ${ }^{41}$, les réticences des différents énonciateurs envers la voix de l'«opinion» invitent également à la vigilance dans l'ordre laïque. C'est d'abord une rumeur sur Félix, blessé en protégeant Olivier, qui est disqualifiée par l'épistolière : "On prétend qu'il était fier de cette blessure : pour moi, je n'en crois rien ${ }^{42}$.» Cette fierté est à l'évidence incompatible avec l'ethos des deux amis. Plus tard, sous la plume du subdélégué Aubert, c'est la fin des aventures de Félix qui se dissipe dans les brumes de l'opinion : "Sa fin eût été malheureuse, si l'amour ne l'eût secouru. La fille du geôlier prit de la passion pour lui, et facilita son évasion. Si cela n'est pas vrai, c'est du moins l'opinion publique ${ }^{43}$.» Le romanesque de la péripétie entraîne une réserve du subdélégué et la désignation d'une instance collective (à la différence des témoins nommément cités) pourvoyeuse de croyances, mais peu fiable. Dans Madame de La Carlière, cette critique devient centrale. Desroches est un exemple «des jugements inconsidérés des hommes ${ }^{44}$ ». Avant son mariage avec Mme de La Carlière, l'opinion l'a condamné pour avoir quitté l'état ecclésiastique, puis celui de magistrat. Dans le second cas, après qu'un condamné effectivement coupable lui a démontré qu’il ne pouvait en réalité pas en avoir la certitude, il a préféré renoncer au risque d'être "souillé du sang de l'innocent». "C'est qu'on ignore ces choses-là ", confesse l'interlocuteur fictif du conte qui avait, avec l'opinion générale, condamné Desroches. Et le «conteur» poursuit :

- C'est qu'il faut se taire quand on ignore.

- Mais pour se taire, il faut se méfier.

— Et quel inconvénient à se méfier?

39. Les Deux Amis de Bourbonne, ouvr. cité, p. 449.

40. Ibid., p. 449 et "préface-annexe» de La Religieuse, ouvr. cité, p. 408.

4I. P. Chartier, "Parole et mystification», art. cité, p. 247 et 248.

42. Les Deux Amis de Bourbonne, ouvr. cité, p. 439-440.

43. Ibid., p. 446.

44. Diderot, Madame de La Carlière, dans Contes et romans, ouvr. cité, p. 520. 
- De refuser de la croyance à vingt personnes qu'on estime, en faveur d'un homme qu'on ne connaît pas ${ }^{45}$.

Ce refus de croyance est pourtant, à lire le conte, une hygiène intellectuelle qui évite de porter un jugement injuste fondé sur l'opinion des «impertinents jaseurs», de la «foule imbécile», du "bavardage du présent ${ }^{46}$ ». Dans le Supplément au voyage de Bougainville, le jugement public apparait d'ailleurs comme l'auxiliaire des croyances religieuses et d'une loi civile inique :

- L'Aumonier : Les coupables qui échappent à la sévérité des lois, sont châtiés par le blâme général.

- Orou : C'est-à-dire que la justice s'exerce par le défaut de sens commun de toute la nation, et que c'est la folie de l'opinion qui supplée aux lois ${ }^{47}$.

La tyrannie de l'opinion ${ }^{48}$ prend le relais de l'influence des «opinions religieuses» : dans les deux cas, Diderot dénonce l'influence des croyances sur le jugement. Le conte en illustre aussi la versatilité, dont il démonte les mécanismes : après la séparation de Desroches et de Mme de La Carlière, celle-ci est d'abord unanimement jugée «folle à enfermer", puis, progressivement, sans plus de fondement, l'opinion passant d'un extrême à l'autre fait de Desroches un monstre, un misérable, un assassin — en attendant que le jugement s'inverse à nouveau.

La fin du conte prend explicitement le parti de Desroches, annonçant la critique par le Supplément au voyage de Bougainville de la fidélité sexuelle rigoureuse qu' exigeait Mme de La Carlière. L'ensemble de la scène du serment par lequel Desroches s'engage à cette intransigeante fidélité introduit cependant un degré supplémentaire de complexité. La croyance y est à nouveau sollicitée, comme confiance, comme crédit donné à la parole d'autrui. Le serment prend place dans un moment paroxystique dont la description renvoie à l'émotion théâtrale. Mme de La Carlière vient de prononcer un grand discours exigeant une fidélité absolue, Desroches lui promet ce qu'elle veut, les amis présents fondent en larmes :

C'est l'effet de ce qui est honnête, de ne laisser à une grande assemblée qu'une pensée et qu'une âme. Comme on s'estime, comme on s'aime dans ces moments! Par exemple, que l'humanité est belle au spectacle! Pourquoi faut-il qu'on se sépare si vite! Les

45. Ibid., p. 52I.

46. Ibid., p. 520,533 et 537 .

47. Diderot, Supplément au voyage de Bougainville, ouvr. cité, p. 558.

48. Cette opinion n'est évidemment pas encore l'«expression de la société civile» (Diderot, Contes et romans, ouvr. cité, p. I062, note I de la p. 446), mais plutôt la voix de la rumeur, fondée sur des préjugés. 
hommes sont si bons et si heureux lorsque l'honnête réunit leurs suffrages, les confond, les rend uns ${ }^{49}$.

Ce moment peut se lire sérieusement, comme la description d'une émotion sincère et utile. Le paroxysme se poursuit par une laïcisation de l'engagement mutuel des époux et un redoublement d'enthousiasme : "Chevalier, dit Mme de La Carlière, je ne vous crois pas encore, mais bientôt je vous croirai ${ }^{5 \circ} . . . »$ Pour ce faire, elle exige un serment laïque, prêté devant l'assemblée des amis, engagement d'un plus grand poids que la cérémonie religieuse à venir, conventionnelle, galvaudée, éloignée des croyances des protagonistes $^{\text {sI }}$. Chaque participant en sera le garant et devra abandonner celui qui ne tiendrait pas parole : "À l'instant, le salon retentit de cris mêlés : Je promets, je permets, je consens, nous le jurons... et au milieu de ce tumulte délicieux, le chevalier qui avait jeté ses bras autour de Mme de La Carlière la baisait sur le front, sur les yeux, sur les joues ${ }^{52}$." La croyance comme confiance en la parole d'autrui se fonde sur une croyance comprise comme adhésion à une valeur, laïcisée par la forme même de la cérémonie : la fidélité sexuelle ne relève plus des opinions religieuses, elle s'est déplacée dans un univers strictement humain.

L'ensemble du passage, porté par une émotion paroxystique, semble écrit de façon à entraîner le lecteur dans ce mouvement d'adhésion. Lorsque l'interlocuteur du conte se souvient d'une "parodie bien comique" du discours de Mme de La Carlière, le narrateur réagit avec vivacité :

Et cela ne suffirait pas à rencogner un homme au fond d'une forêt, loin de toute cette décente canaille pour laquelle il n'y a rien de sacré? J'irai, cela finira par là, rien n'est plus sûr, j'irai ${ }^{33}$.

Mais lorsque le serment débouche sur des conséquences catastrophiques, l'adhésion à la grande scène se fissure. Les témoins la réinterprètent différemment :

[S]i nous eussions vu sa sublime momerie du même œil que le public et la comtesse ${ }^{54}$, rien de ce qui nous désole à présent ne serait arrivé... C'est que les choses d'un certain

49. Diderot, Madame de La Carlière, ouvr. cité, p. 525.

50. Ibid.

5I. «La présence de Dieu est moins redoutable pour nous que le jugement de nos semblables» énonce Mme de La Carlière (ibid., p. 525-526).

52. Ibid., p. 526.

53. Ibid., p. 525 .

54. Il s'agit sans doute de «l'auteur» de la parodie du discours de Mme de La Carlière. 
appareil nous en imposent, et que nous nous laissons aller à une sotte admiration lorsqu'il n'y aurait qu’à hausser les épaules et riress...

Ces lignes sont une nouvelle illustration de la versatilité de l'opinion et un contrepoint critique de la scène comme du commentaire du narrateur, dont elles soulignent l'étrangeté partielle : à la différence des témoins, celui qui raconte connaît au moment du récit les conséquences désastreuses du serment. Son enthousiasme pour le caractère «sacré» de l'engagement pris peut a posteriori surprendre, quand on l'entend critiquer l'intransigeance de Mme de La Carlière, lorsqu'on le voit imaginer une histoire alternative dans laquelle les époux se seraient réconciliés, auraient échangé de nouveaux serments, bientôt rompus sans conséquence tragique : chronique, en somme, d'une infidélité ordinaire et souriante, ne se prêtant plus à la ferveur du serment, mais échappant aux catastrophes que celui-ci provoque; histoire moins sublime et plus humaine.

Madame de La Carlière accomplit donc, sur un plan différent, les trois opérations rencontrées dans Les Deux Amis de Bourbonne: la critique d'un jugement fondé sur de fausses croyances, ici portées par l'opinion, ainsi que du mécanisme d'assentiment à ces jugements ("ne les croyez point", met en garde le conteur ${ }^{56}$ ); la construction d'un effet d'adhésion (la scène du serment au moment où elle est racontée) et la déconstruction de cet effet, par le renversement du discours des témoins (lui-même susceptible d'être critiqué), puis à travers la conclusion du conte. Il ne s'agit plus ici de croyances attachées à des faits, mais à une valeur. La conclusion critique sur cette valeur qui soutenait le serment n'a pas pour conséquence d'annuler la puissance d'émotion de la scène qui le représentait. La lucidité critique n'interdit pas le paroxysme sensible : elle en représente plutôt le nécessaire contrepoint.

Dans Mystification, Diderot adopte devant Mlle Dornet une ironie souriante et sans agressivité lorsqu'elle évoque le savoir et les pouvoirs du «soi-disant médecin turc» envoyé pour récupérer les portraits de Galitzine : «vous êtes tolérant» reconnaît-elle lorsque Diderot lui déconseille d'évoquer ses «enfantillages» devant l'intransigeant Naigeon ${ }^{57}$. Pour la convaincre que les portraits de son ancien amant mettent sa santé en péril, l'imposteur se fonde sur «la vieille doctrine d'Épicure» : des simulacres viendraient s'attacher à la rétine ${ }^{58}$. Sans reprendre à son compte les doctrines de Bonvalet-

55. Ibid., p. 532.

56. Ibid., p. 537.

57. Diderot, Mystification, ouvr. cité, p. 433.

58. Ibid., p. 427-428. 
Desbrosses, Diderot juge à son tour «qu'on n'a rien de mieux à faire que de se détacher de tous les objets qui réveillent en nous un souvenir fâcheux ${ }^{59}$ ». Cette tolérance et l'accord donné à la conclusion des raisonnements fantaisistes du faux-médecin tiennent-ils seulement au rôle de Diderot dans la mystification? Le récit place le personnage du philosophe dans une situation paradoxale, puisqu'il représente aux yeux de Mlle Dornet un incrédule qui doit par définition chercher à démasquer un imposteur, quand le lecteur sait qu'il est en réalité le commanditaire de l'imposture. Lopposition entre Diderot et Naigeon peut donc avoir une motivation narrative : le premier n'entend pas que sa dupe soit désabusée par son disciple. La bienveillante et peu insistante démystification suggérée par Diderot est encore un temps de la mystification - structure qui, si elle devait fonctionner comme un prisme de lecture pour Les Deux Amis de Bourbonne ou La Religieuse, constituerait un degré supplémentaire de complexité.

Ici, cependant, le lecteur est d'emblée placé du côté des "complices» (Diderot, Bonvalet-Desbrosses et Mme Therbouche). Il perçoit la façon dont l'imposteur suscite la croyance de sa dupe : l'analyse du mécanisme de la croyance se déroule de façon plaisante, parfois comique. L'effet de croyance est par exemple obtenu par un travail de confirmation des attentes de la dupe : "Je suis donc malade? Dieu soit loué!», s’exclame Mlle Dornet lorsque le faux médecin le lui affirme ${ }^{60}$. L'imposteur commence par s'ériger en miroir complaisant renvoyant à la dupe l'image qu' elle veut avoir d'ellemême. Il fonde son pouvoir sur cette gratification narcissique. Lorsque Desbrosses décrit Mlle Dornet comme «une femme à talent», le corps de la jeune femme se met en mouvement pour fixer cette image valorisante : "Cependant Mlle Dornet, flattée du mot de femme à talent, faisait tout ce qu'il fallait pour que le docteur n'en démordît pas; elle ne dansait pas, mais elle s'en donnait les airs ${ }^{6 \mathrm{I}}$." La dupe commence à avoir besoin de l'imposteur pour faire durer l'illusion qui la flatte. Bientôt, c'est elle-même qui confirme ses jugements en les faisant devenir réalité. Desbrosses a trouvé sur son visage des traces de tristesse : "La demoiselle Dornet était si curieuse de faire dire la vérité au docteur, qu'à mesure qu'il parlait et que Mme Therbouche la regardait,

59. Ibid., p. 434. Par une stimulante coïncidence, le moyen choisi par Diderot pour récupérer les portraits fait écho à l'une des premières «expériences» sollicitées par Hume pour expliquer la vivacité qui caractérise la croyance : «à l'apparition du portrait d'un ami absent, l'idée que nous avons de lui est évidemment avivée par la ressemblance, et [...] toute passion occasionnée par cette idée, qu'elle soit de joie ou de chagrin, acquiert une force et une vigueur nouvelle» (Hume, Traité de la nature humaine, ouvr. cité, p. I64).

6o. Diderot, Mystification, ouvr. cité, p. 420.

6I. Ibid., p. 42 I. 
son visage prenait l'expression de la tristesse ${ }^{62}$.» La gratification narcissique a accompli sa tâche, c'est désormais la dupe elle-même qui désire que ce qu'on lui dit soit la vérité, qui est prête à opérer les ajustements nécessaires pour qu'il en soit ainsi. Le sourire de Mlle Dornet lorsqu'elle lit ce que l'imposteur prétend avoir découvert dans la paume de sa main en témoigne : la croyance est désormais associée à un principe de plaisir qui, on le conçoit, n'invite guère à la méfiance ${ }^{63}$. La touche d'exotisme apportée par la prétendue origine turque du faux médecin joue sur la curiosité ${ }^{64}$, culturelle et sexuelle ( Cela doit être singulier, un homme circoncis $\left.{ }^{65} \gg\right)$; elle permet d'introduire les croyances les plus manifestement superstitieuses en les faisant passer pour un savoir venu d'ailleurs : opération qui, elle, se fonde sur la disposition à la crédulité évoquée par Diderot au début du conte. La manipulation de la peur de la dupe n'intervient qu'après l'ensemble de ces préparatifs : lorsque Desbrosses illustre par des exemples de plus en plus inquiétants l'influence néfaste des portraits, des objets et des lieux associés à un ancien amour, Mlle Dornet lui a déjà accordé sa croyance.

Desbrosses, assuré de son pouvoir, peut se permettre d'adresser à Mme Therbouche, et indirectement au lecteur, des clins d'œil qui dénoncent la mystification au moment où elle se déploie. La dupe et la complice entendent la même phrase, mais ne lui prêtent pas la même signification. L'une est abusée, l'autre voit dans les mots du mensonge sa révélation ironique. Ainsi lorsque Mlle Dornet s'étonne que le médecin turc puisse dire la bonne aventure, métier qu'elle croyait être celui d'un "fripon", Desbrosses en convient et répond par une plaisante diversion que seule une dupe déjà convaincue peut accepter comme un argument sérieux : "C'est assez l'ordinaire; mais un fripon n'empêche pas qu'il n'y ait d'honnêtes gens, non plus qu'un charlatan qu'il n'y ait de vrais médecins ${ }^{66}$." L'énoncé a deux destinataires : Mme Therbouche qui perçoit la moquerie («Rien n'est plus juste», répond-elle) et Mlle Dornet, qui ne la perçoit pas et se laisse mystifier : «regardez donc bien vite ma main », réclame-telle. Peu après, Desbrosses, qui s'est donc déclaré chiromancien, répond à Mlle Dornet évoquant la crainte que ce pouvoir doit susciter : «Et voilà

62. Ibid., p. 422.

63. «Elle ouvre le papier, elle lit, et en lisant elle souriait et disait : Ma foi, cela est vrai... Cela l'est encore... Mais cela est prodigieux... Comment est-il possible qu'on ait sa vie écrite dans sa main?...» (Ibid., p. 424)

64. Cette disposition, plusieurs fois reconnue, joue évidemment un rôle crucial dans le dispositif du conte.

65. Ibid., p. 423.

66. Ibid., p. 424, où se trouvent également les deux citations suivantes. 
précisément pourquoi les vrais chiromanciens s'en cachent ${ }^{67}$.» La phrase peut être reçue «sérieusement" par la dupe, flattée que le médecin ne s'en cache plus devant elle. Par la contradiction entre l'énoncé et l'énonciation, elle est aussi plaisante et ironique : si le vrai chiromancien s'en cache, celui qui ne s'en cache pas est donc un imposteur. La phrase de Desbrosses justifiant sa volonté de ne rencontrer «Diderot» qu'un quart d'heure avant son départ ("C'est que je ne reste point dans un endroit quand j'y suis connu $^{68} "$ ) se prête encore à une double lecture : elle contribue à l'aura mystérieuse d'un imposteur que la dupe désire de plus en plus considérer comme un sorcier ${ }^{69}$; elle indique également la précipitation avec laquelle le faux médecin doit partir lorsque son manège est dévoilé. Ce procédé, où se perçoit la relation qu'entretiennent mystification et persiflage ${ }^{70}$, est aussi la représentation du jeu de Diderot conteur avec son lecteur : celui-ci est amené à occuper en même temps des positions comparables à celle de la dupe et de la complice, à accorder sa croyance et à percevoir les indices invitant à la méfiance ${ }^{71}$.

Mais comment justifier l'influence des portraits, comme celle des objets et des lieux qui se rapportent à un être autrefois aimé? Par la doctrine d'Épicure, on l'a vu. Plus largement, l'imposteur se fonde sur une anthropologie hostile au dualisme et sensible aux mouvements discrets du désir :

Mme Therbouche : Et puis est-il possible qu'il ne nous reste pas de nos goûts une pente secrète?

Desbrosses : N'en doutez pas. Nous la suivons d'abord sans le sentir; sa force s'accroît en nous sourdement, tant et si bien qu'elle finit à la longue par nous entrainer avec une violence à laquelle on ne résiste plus. La théologie a voulu s'en mêler; mais

67. Ibid., p. 425 .

68. Ibid.

69. «Desbrosses ne demandait pas mieux que de s'avouer sorcier pour faire plaisir à la belle dame» lit-on plus tard (ibid., p. 43I).

70. Voir P. Chartier, Théorie du persiflage, Paris, PUF, 2005; et "Diderot, ou le rire mystificateur", art. cité.

7I. Selon J.-C. Rebejkow, cette relation caractérise la mystification diderotienne elle-même, qui se distinguerait ainsi de la tromperie : «Dans la mystification, la relation est duelle: il n'y a pas de relation unilatérale, la personne mystifiée n'est pas "méprisée", puisqu'elle est avertie de la mystification en cours par des indices intermédiaires. Alors que dans la tromperie, à la différence de la mystification, il y a une relation univoque : le trompé ne doit pas être désabusé, il est passif, "réifié"; le trompeur veut le priver de toute réflexion critique." ("À propos de Mystification : l'ironie de Diderot", The Romanic Review, vol. 89, $\mathrm{n}^{\circ}$ 4, I998, p. 507-521, p. 512.) Il nous semble cependant que les indices intermédiaires ne sont ici nullement adressés à la dupe, mais au complice (et au lecteur) : la relation duelle caractérise moins la mystification comme pratique sociale que le réinvestissement littéraire de cette pratique. De même, la «double lecture» analysée par J.-C. Rebejkow est sollicitée par les textes, mais elle n'est pas celle de la dupe dans une mystification réelle. 
affaire d'organisation, effet naturel, affaire de médecine. On devient triste sans raison, à ce qu'on croit, premier symptôme. L'ennui nous gagne; nous cherchons à nous dissiper, nous ne le pouvons, partout il nous manque quelque chose ${ }^{72}$.

"Mais affaire d'organisation, effet naturel, affaire de médecine» : si les réponses de l'imposteur et sa description des "sympathies » sont fantaisistes, la question posée et les prémisses sur lesquelles Desbrosses se fonde sont aussi celles de Diderot ${ }^{73}$. De même pour la syntaxe cumulative de ce fragment : les voix du philosophe et de l'aventurier s'entremêlent. L'imposteur se fonde sur des observations physiologiques qui occupent aussi le matérialiste. Les meubles, les bijoux, les lettres, les portraits, agissent sur le corps : «la digestion est plus ou moins dérangée, le sommeil interrompu; on fait des rêves, on a des palpitations; l'imagination s'échauffe, le sens se brûle, le tempérament se détruit, on tombe dans un état misérable ${ }^{74}$ ». Chez l'imposteur, les croyances superstitieuses vont venir combler une connaissance de l'homme lacunaire qui ne peut plus s'appuyer sur les anciennes évidences. Elles apportent des réponses erronées à de véritables questions. À ce titre, le manipulateur de la croyance est l'autre du philosophe et du conteur. Par son talent pour emporter l'adhésion, son art de la mise en scène, par l'anthropologie même qui fonde son discours sur le pouvoir des objets, il est aussi leur double.

La «tolérance» de Diderot à la fin du conte n'est donc pas seulement un temps de la mystification. Le conteur déconstruit les effets de croyance qu'il produit : cela ne revient pas à abolir toute croyance, mais à faire fonctionner celle-ci conjointement à un principe de lucidité qui ne s'y substitue pas pour autant. Il faut en partie croire à l'histoire d'Olivier et

72. Diderot, Mystification, ouvr. cité, p. 427-428.

73. Nous rejoignons une interprétation du conte proposée par M. Delon : «Le conte est une nouvelle version d'un Portrait du philosophe en mystificateur que Diderot peaufine, d'œuvre en œuvre. Le philosophe imagine le stratagème, l'aventurier le réalise. Le philosophe s'interroge sur les liens du physique et du moral, sur les déterminismes qui donnent à un objet, à un décor, à un paysage une signification psychologique particulière. L'aventurier se réclame de sympathies mystérieuses et d'influences occultes. On s'est souvent demandé comment le siècle des Lumières pouvait être celui de l'illuminisme, du mesmérisme et de toutes les doctrines fumeuses dont vivaient des aventuriers de haut vol et dont les victimes étaient les princes et les puissants, à travers toute l'Europe. L'interrogation sur la continuité du matériel et du moral qui met en cause le dualisme du rationalisme classique cherche des arguments dans les observations scientifiques et débouche alors sur le matérialisme de Diderot, puis des idéologues, ou bien se tourne vers les croyances archaïques et nourrit les impostures de Casanova, de Cagliostro, de Mesmer et de tant d'autres. Naigeon, qui apparait à la fin du conte, sert de repoussoir à Diderot. Son rationalisme rigoureux récuse les aventures de l'esprit qui justement passionnent le philosophe, intellectuellement et esthétiquement. (Dans Diderot, Contes et nouvelles, ouvr. cité, notice de Mystification, p. I040.)

74. Diderot, Mystification, ouvr. cité, p. 426. 
Félix pour que la conclusion pessimiste du conte produise son effet; mais cette croyance ne se confond pas avec un leurre véritable. L'immersion fictionnelle ${ }^{75}$ n'est pas une hallucination, le lecteur n'est pas une dupe - quoique le péritexte puisse faire de la puissance mystificatrice du texte une composante de sa lecture. Le serment de fidélité dans Madame de La Carlière est, au moment où il est prêté, un paroxysme émotionnel pensé par comparaison avec le théâtre; il suppose une adhésion à la valeur et aux idées qu'il engage. Cette émotion est partagée par le lecteur au moment où il découvre la scène, quoique la suite du texte le pousse à déconstruire le mécanisme d'adhésion qui l'a fait participer à cette cérémonie aux conséquences funestes. Est-ce à dire, a posteriori, qu'il n'y a rien à conserver de l'émotion même? Que le lecteur a été simplement berné par un «appareil» imposant? Le texte de Diderot est plus complexe : la critique idéologique de la fidélité sexuelle intransigeante est livrée par la fin du conte, mais celle du serment à proprement parler est confiée à des personnages inquiets de leur propre réputation et illustrant à leur tour la versatilité de l'opinion : la critique de ce moment paroxystique et profondément dramaturgique est déléguée à des personnages qui ne donnent pas nécessairement le dernier mot du conte, elle n'est pas le fait d'une instance dotée d'une «autorité» narrative ou morale supérieure. Dans Mystification, si la dupe, sa croyance et les superstitions sollicitées par l'imposteur prêtent à sourire, le philosophe-commanditaire et le manipulateur-acteur sont aussi des instances complémentaires de la mystification dont le conte constitue l'analyse. Leurs voix peuvent même fugitivement se mêler.

La croyance, à lire ce conte, relève d'une logique du désir, jusque dans ses manifestations sexuelles. Mlle Dornet se prête volontiers aux investigations de Desbrosses qui prétend avoir besoin de la toucher pour comprendre sa maladie. Mme Therbouche ironise : «Au moins docteur, si vous ne rencontrez pas, ce ne sera pas la faute de mademoiselle, elle s'est prêtée de bonne grâce à vos observations ${ }^{76}$.» La scène peut illustrer une idée que l'on trouve développée ailleurs : une femme inquiète pour sa santé oublie la pudeur. Elle fait aussi écho à la curiosité de Mlle Dornet pour le sexe circoncis du prétendu Turc. Après le départ de l'imposteur, le dialogue se poursuit entre Mlle Dornet et Mme Therbouche :

Mme Therbouche : [...] il faut que vous l'ayez singulièrement intéressé pour se lâcher comme il l'a fait.

Mlle Dornet : Il aime les femmes.

75. Voir J.-M. Schaeffer, Pourquoi la fiction?, ouvr. cité.

76. Diderot, Mystification, ouvr. cité, p. 42I. 
Mme Therbouche : Les belles femmes, à la folie.

Mlle Dornet : Vous me l'amènerez?

[...]

Mme Therbouche : Vous n'oublierez pas que c'est contre mon gré, que c'est vous qui l'avez voulu?

Mlle Dornet : Oui, oui c'est moi qui l'aurai voulu, qui le veux ${ }^{77}$.

Le dialogue montre l'habileté de Mme Therbouche qui feint de mettre en garde la dupe pour lui faire assumer l'entière responsabilité de la prochaine rencontre. Il met en scène le succès complet de la mystification, Mlle Dornet lui donnant désormais son plein assentiment. Il montre en outre un certain appel de la croyance : le manipulateur parvient à ses fins lorsqu'il répond à un désir. Ce besoin de croire au risque de l'erreur est redoublé par le désir plus nettement sexualisé qui transparaît dans le passage sur l'amour des femmes : le dialogue représente aussi le succès d'un pouvoir de séduction qui excède les affabulations de l'imposteur. L'assentiment au désir de croire est doublé d'une aspiration érotique à peine voilée. Si le conteur ne place jamais le lecteur dans la position de Mlle Dornet, la dupe réellement abusée, on conçoit que la critique des croyances religieuses, superstitieuses ou laïques, celles qui relèvent de la rumeur et de l'opinion, ne s'accompagne pas d'une démystification pure et simple de la croyance entendue comme disposition ou comme aspiration humaine. Diderot en déconstruit les mécanismes pour le plaisir de l'intelligence, pour les vertus de la lucidité. Le conteur ne disqualifie pas pour autant cette croyance qui est aussi la condition d'une certaine adhésion au récit et du paroxysme émotionnel, opération complexe qui engage le plaisir littéraire, l'efficacité des fictions et les mouvements du désir.

77. Ibid., p. 432-433. 